\title{
MODELLING MIXED CONVECTION IN LAMINAR PIPE FLOW
}

\author{
M.H. BUSCHMANN ${ }^{1}$, L. COLLA ${ }^{2} \&$ L. FEDELE ${ }^{2}$ \\ ${ }^{1}$ Institut für Luft- und Kältetechnik Dresden, Dresden, Germany. \\ ${ }^{2}$ National Research Council - Institute of Construction Technologies, Padova, Italy.
}

\begin{abstract}
Laminar pipe flow is becoming an important experimental test case for new high efficiency heat carriers like nano- and ferrofluids. Here, a new scaling approach for mixed convection in laminar pipe flow with constant heat flux is proposed. The model relates the radial temperature gradient at the wall, represented as the local Nusselt number, with local Reynolds, Prandtl, and Grashof numbers. The proposed scaling approach is successfully employed to collapse data from different test rigs with horizontally oriented pipes and operated with water. Influences of differing strengths following from free convection are gathered with the new scaling. Moreover, the new scaling approach is successfully utilised to value experimentally obtained heat transfer data of nanofluid flow. In this regard, the impact of nanoparticles, namely the suppression of heat transfer in mixed convection, is experimentally shown and theoretically analysed. Finally, the influence of pipe orientation (vertical / horizontal) is discussed.

Keywords: free convection, modelling, nanofluids, pipe flow.
\end{abstract}

\section{INTRODUCTION}

Nano- and ferrofluids are a new option to enhance heat transfer. Worldwide, several test rigs are currently being operated to validate the qualities of these special fluids. Mostly, thermally developing laminar pipe flow is employed for these investigations. The advantage of this type of flow is that it is analytically describable based on first principles alone. Unfortunately, these analytical solutions are only correct if several physical effects are ignored. Among them is buoyancy, which occurs in nearly all real world flows.

In pipe flow, buoyancy follows from thermal instabilities caused by radial or axial temperature gradients. In a horizontal pipe, for example, a secondary flow is generated by these instabilities. Two axially oriented counter-rotating vortices develop. Heat transfer is now coined by mixed convection. Thereby, mixed indicates that the heat transfer is partly due to thermal conduction and partly due to free convection. The latter component causes a significantly increased Nusselt number which is well above the forecasted value of the analytical solution.

Nano- and ferrofluids are suspensions. Basically, they consist of a basefluid like water, alcohol, or oil, and nanoparticles. The size of the suspended nanoparticles ranges from $10 \mathrm{~nm}$ to $100 \mathrm{~nm}$. Additionally, dispersant agents or surfactants are added to stabilise the liquid against agglomeration, aggregation, and therewith against sedimentation. Basefluids usually have a low thermal conductivity. By adding nanoparticles which have a significantly higher thermal conductivity, the effective thermal conductivity of the suspension is increased. Several experimental investigations [1] have shown that this goal can indeed be achieved.

Disadvantageously, nanoparticles also cause unexpected and unwanted effects. While thermal conductivity is usually increased moderately, viscosity is sometimes increased 
significantly. This of course lowers the Reynolds number and, even worse, boosts the required pumping power. Other negative effects like aging and wall-nanoparticle interaction are currently under investigation.

Modelling, including numerical simulation, of such complicated flow situations is not completely understood [2]. Therefore, standardised experiments are still key for understanding nanofluids. With respect to nano- and ferrofluids, thermally developing laminar pipe flow is becoming such a test case [3]. However, the flow situation occurring in these seemingly simple experiments has to be validated too.

Experimental investigations show that free convection in pipe flow is significantly affected by nanoparticles [4]. The development of secondary flow structures supporting conductive heat transfer is obviously suppressed. Unfortunately, even with classical single-phase fluids like pure water no suitable theory exists to describe the development of secondary motion. Thus, it becomes quite complicated to distinguish between effects following from secondary motion or from Brownian and thermophoretic diffusion of nanoparticles and the basic heat transfer connected with the thermal conductivity of the basefluid. Especially Brownian and thermophoretic diffusion may affect local heat transfer in an antagonistic way [5].

Altogether this indicates an urgent need for improved models for laminar pipe flow affected by free convection. The scope of this paper is to provide such an approach and to discuss its applicability with respect to laminar nanofluid flow in straight pipes.

\section{ANALYTICAL APPROACH}

For laminar pipe flow, two boundary conditions have to be distinguished. The first is the uniform surface temperature (UST) condition (Dirichlet boundary condition). An analytical approach for this case was already provided by Graetz [6] in 1883 and later improved by Nusselt [7]. However, most test rigs do not work with this boundary condition. They rather employ a uniform heat flux (UHF) boundary condition (Neumann boundary condition) generated either by a heater wrapped around the pipe or by heating the pipe utilising its ohmic resistance. Siegel et al. [8] and Hsu [9], among others, provided an analytical solution for this case eqn (1). Similar as for the UST-case, the solution is a series expansion in $x^{+}$the non-dimensionalised axial coordinate of the pipe.

$$
N u\left(x^{+}\right)=\left[\frac{48}{11}+\frac{1}{2} \sum_{n=1}^{\infty} A_{n} e^{-2 \beta_{n}^{2} x^{+}}\right] ; \quad x^{+}=\frac{x}{d_{i}} \frac{1}{\operatorname{RePr}}
$$

In (1) $d_{i}$ denotes the inner diameter of the pipe. Nusselt, Reynolds, and Prandtl number are represented by $\mathrm{Nu}, \operatorname{Re}$, and $\mathrm{Pr}$, respectively. For $x^{+}$approaching infinity the Nusselt number becomes $48 / 11(\approx 4.364)$. Values for coefficients $A_{n}$ and powers $\beta_{n}$ up to 20 th were first predicted with sufficient accuracy by Hsu [9].

In Fig. 1, the upper line shows experimental results of laminar pipe flow from [10]. Working fluid is water. For small $x^{+}$-values, the agreement with the analytical solution (1) is obvious. Above a $x^{+}$-value of about $10^{-2}$, secondary motion becomes significantly stronger. The developing pair of counter-rotating vortices transports fluid from the hot inner pipe wall toward the cooler core of the flow. Heat transfer is enhanced and the increasing Nusselt number departs more and more from the analytical solution. Moreover, data of different experiments no longer collapse within one curve. The flow is now characterised by the term mixed convection. The situation is different for the vertical pipe by Rea et al. [11]. No departure from the 

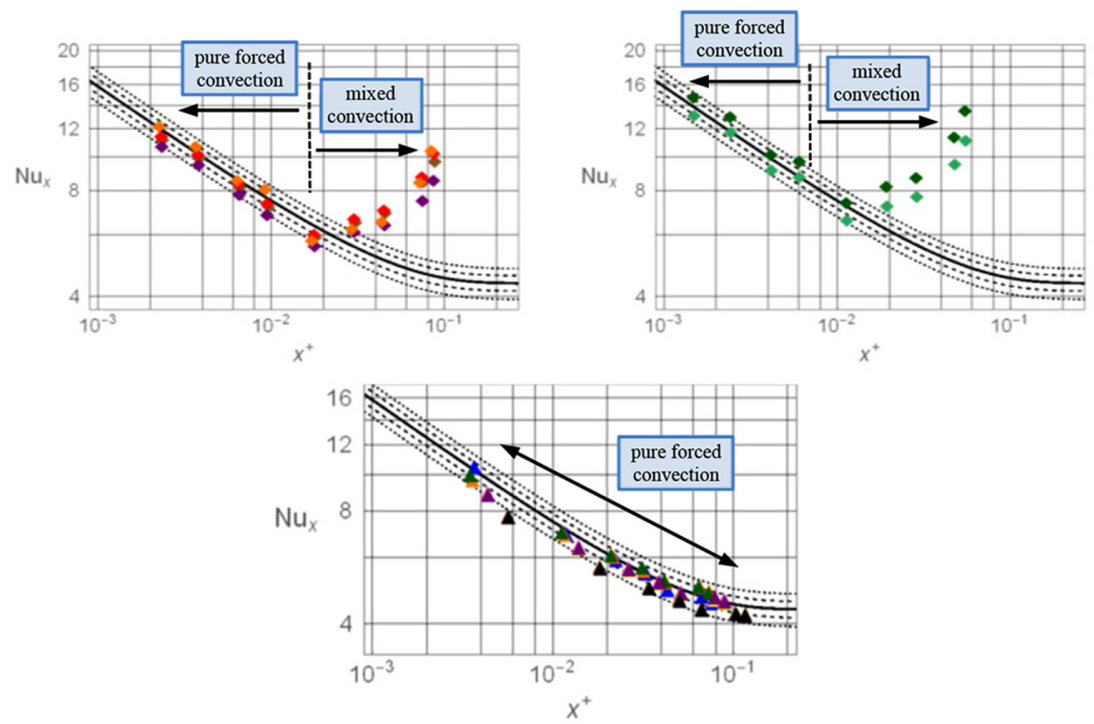

Figure 1: Typical Nusselt number distributions along the mean flow axis of a laminar flow pipe in the entrance region operated with classical single-phase liquids. Upper line data from Buschmann [10]. Lower line data from Rea et al. [11]. Full curves indicates eqn (1), dashed curves a departure of $\pm 5 \%$, and dotted curves of $\pm 10 \%$.

theory by Siegel et al. [8] and Hsu [9] is recognised and, accordingly, significant mixed convection is observed.

No general analytical solution is known so far for mixed laminar pipe flow with uniform heat flux boundary condition. Approaches provided by Morton [12] and van Dyke [13] are limited in their applicability [4]. In general, an analytical solution should follow the form

$$
N u_{x, \varphi}=f\left(\operatorname{Re}, \operatorname{Pr}, G r, \frac{x}{d_{i}}, \varphi\right)
$$

Here, $G r$ denotes the Grashof number, the ratio of the buoyancy to viscous force acting on a fluid. The variable $\varphi$ is the peripheral angle.

An analytical approach according to (2) was proposed by Hieber and Sreenivasan [14] for UST boundary condition. The scope of the present study is to investigate if this approach is also applicable for laminar mixed pipe flow with UHF boundary condition.

With their approach, Hieber and Sreenivasan [14] discriminated between four regions. In the first region, buoyancy is negligibly small compared with forced convection and can be neglected. Experimental data follow the exact solution without free convection. In the case of constant heat flux at the wall, such a region exists as well (Fig. 1 below $x^{+}$-value of about $10^{-2}$ ). The second region is characterised by an increasing free convection and the experimental data start to depart from the analytical solution without free convection. Again, such a region exists in the case discussed here (Fig. 1 above $x^{+}$-value of $10^{-2}$ ). In flow region three, the core region of the flow is heated to the point that free convection starts to collapse. The Nusselt number does not increase any further. The examples shown in Fig. 1 do not show such a region, but many several experiments indicate a region three [4]. After the temperature profile 
has homogenised, forced convection becomes dominant again. Nusselt numbers decrease and finally reach the analytical approach without free convection. Such a region is seldom seen in experiments mainly because pipes are too short. Anyway, region four is not relevant for the analysis we carried out here.

In the following, the study will focus on the second region. It is assumed that free convection exists all along the first region, but it is so weak that it cannot be resolved employing the usual experimental equipment. Meaning measuring temperatures and calculating Nusselt numbers as usually is done is not sufficient to show these effects.

Hieber and Sreenivasan [14] propose for the second region a scaling based on the quantity:

$$
\varepsilon_{R 2}=\frac{2 x}{d_{i}} \frac{1}{\operatorname{Re}} \frac{G r^{1 / 4}}{\operatorname{Pr}^{3 / 4}}
$$

Note that (eqn 1) demands that $R e, P r, G r$ and the ratio $x / d_{i}$ are considered in an approach integrated over the circumference of the pipe. Hieber and Sreenivasan [14] reduce this to one single parameter which contains all of these similarity numbers. However, the discrete influence of each of these similarity numbers is still considered.

For the temperature distribution along the pipe axis Hieber and Sreenivasan [14] derived the following correlation:

$$
\Phi\left(\varepsilon_{R 2}\right)=\left(\frac{\mu_{\mathrm{w}}}{\mu_{\mathrm{b}}}\right)^{0.14}\left(\begin{array}{ll}
T_{b} & T_{i n} \\
T_{w} & T_{i n}
\end{array}\right)_{\text {exp }}
$$

Here, $T_{b}$ denotes the "mixing-cup" or bulk temperature, $T_{w}$ is the wall temperature, and $T_{\text {in }}$ is the inlet temperature at the beginning of heating. The influence of the temperature dependent viscosity is included by the power of the viscosity ratio $\mu_{w} / \mu_{b}$. The most important difference of (eqn 3) compared to other approaches, e.g. [12,13], is that the development of $T_{b}$ is indeed considered in dependency on the mean flow axis.

Based on physical consideration and considerable algebra, Hieber and Sreenivasan [14] proposed a series expansion up to the 6th order for $\Phi\left(\varepsilon_{R 2}\right)$. Here, this series expansion is extended up to 20th order. For that purpose, the system of differential eqn (4) is solved numerically employing MATHEMATICA 10.1.

$$
\begin{aligned}
& f_{n}^{\prime \prime}+h_{n}=0 \\
& h_{n}^{\prime \prime}+\sum_{k=0}^{n} f_{n-k} h_{k}^{\prime}=0
\end{aligned}
$$

For more details with respect to the derivation of system (4), refer to [14].

The function $f$ is related via the stream function $\Psi_{B}$ with the buoyancy induced radial $u_{b}$ and circumferential velocity $v_{b}$ components.

$$
\begin{gathered}
u_{B}=\frac{1}{d_{i} / 2} \frac{\partial \Psi_{B}}{\partial \phi}=\frac{\sqrt{x v / u_{i}}}{d_{i} / 2}\left(\frac{v}{d_{i} / 2}\right)\left(\frac{G r}{\operatorname{Pr}^{2 / 3}}\right)^{1 / 2} \frac{\partial \hat{f}}{\partial \zeta} \\
v_{B}=-\frac{\partial \Psi_{B}}{\partial \mathrm{r}}=\left(\frac{v}{d_{i} / 2}\right)\left(\frac{G r}{P r^{2 / 3}}\right)^{1 / 2} \frac{\partial \hat{f}}{\partial \phi}
\end{gathered}
$$


where

$$
\widehat{f}(\zeta, \phi)=\xi^{3 / 4} f(\eta)
$$

with the angular coordinate $\phi$ and the non-dimensionalised radial coordinate $\zeta$. In (5) the viscosity is denoted by $v$, the axial coordinate by $x$, the inlet velocity at the beginning of the pipe $u_{i}$, and the inner diameter of the pipe is $d_{i}$. So $f$ basically stands for the non-dimensionalised wall-normal velocity distribution in the system of differential equations. In the center of the pipe the boundary conditions are $f(0)=0$ and $f^{\prime}(0)=0$.

Table 1 gives the parameters for the first six orders. The differences between the results by Hieber and Sreenivasan [14] and the values obtained with work are reasonably low. Results for orders $\mathrm{n}=7$ to $\mathrm{n}=12$ are shown in Table 2 and the predicted values of $C_{n}$ in Tables 3 and 4 .

In eqn. (4) the function $h$ represents the non-dimensionalised bulk temperature development. Two additional boundary conditions are needed to solve system (4).

$$
f^{\prime \prime}(\infty)=h(\infty)=0
$$

Table 1: First six terms of series expansion in comparison with Hieber and Sreenivasan [14].

\begin{tabular}{lcccccc}
\hline & $\mathrm{n}=1$ & $\mathrm{n}=2$ & $\mathrm{n}=3$ & $\mathrm{n}=4$ & $\mathrm{n}=5$ & $\mathrm{n}=6$ \\
\hline $\begin{array}{l}f_{n}^{\prime \prime}(0) \\
\text { Ref. [14] }\end{array}$ & 1.16604 & -0.76130 & 0.33136 & -0.12019 & 0.03024 & -0.01195 \\
$f_{n}^{\prime \prime}(0)$ & & & & & & \\
this work & 1.16604 & -0.75821 & 0.32250 & -0.12160 & 0.03885 & -0.01211 \\
difference & $0 \%{ }^{*}$ & $0.009 \%$ & $0.01 \%$ & $-0.564 \%$ & $0.318 \%$ & $0.839 \%$ \\
$h_{n}^{\prime}(0)$ & & & & & & \\
Ref. [14] & -0.50275 & 0.54706 & -0.35717 & 0.18137 & -0.07894 & 0.03093 \\
$\begin{array}{l}h_{n}^{\prime}(0) \\
\text { this work }\end{array}$ & -0.50275 & 0.54658 & -0.35532 & 0.18006 & -0.07829 & 0.03069 \\
difference & $0 \%{ }^{*}$ & $-0.007 \%$ & $-0.037 \%$ & $-0.233 \%$ & $-0.177 \%$ & $-0.752 \%$ \\
\hline
\end{tabular}

*Exact solution without numerical errors.

Table 2: Seventh to twelfth terms of series expansion, this work.

\begin{tabular}{lllllll}
\hline & $\mathrm{n}=7$ & $\mathrm{n}=8$ & $\mathrm{n}=9$ & $\mathrm{n}=10$ & $\mathrm{n}=11$ & $\mathrm{n}=12$ \\
\hline$f_{n}^{\prime \prime}(0)$ & & & & & & \\
this work & $3.42 \times 10^{-3}$ & $-9.72 \times 10^{-4}$ & $2.57 \times 10^{-4}$ & $-6.92 \times 10^{-5}$ & $1.81 \times 10^{-5}$ & $-4.61 \times 10^{-6}$ \\
$h_{n}^{\prime}(0)$ & & & & & \\
this work & $-1.11 \times 10^{-2}$ & $3.81 \times 10^{-3}$ & $-1.24 \times 10^{-3}$ & $3.90 \times 10^{-4}$ & $-1.18 \times 10^{-4}$ & $3.52 \times 10^{-5}$ \\
\hline
\end{tabular}


Table 3: First six calculated values of $C_{n}$ in comparison with Hieber and Sreenivasan [14].

\begin{tabular}{lcccccc}
\hline & $\mathrm{n}=1$ & $\mathrm{n}=2$ & $\mathrm{n}=3$ & $\mathrm{n}=4$ & $\mathrm{n}=5$ & $\mathrm{n}=6$ \\
\hline$C_{n}[14]$ & 0.87052 & -0.47363 & 0.20615 & -0.07851 & 0.02734 & -0.00892 \\
$C_{n}$ this work & 0.87052 & -0.47322 & 0.20508 & -0.07792 & 0.02711 & -0.00886 \\
difference & $0 \%{ }^{*}$ & $0.086 \%$ & $0.519 \%$ & $0.751 \%$ & $0.840 \%$ & $0.673 \%$ \\
\hline
\end{tabular}

${ }^{*}$ Exact solution without numerical errors.

Table 4: Calculated value of $C_{n}$ for seventh to twelfth orders of solution.

\begin{tabular}{ccccccc}
\hline & $\mathrm{n}=7$ & $\mathrm{n}=8$ & $\mathrm{n}=9$ & $\mathrm{n}=10$ & $\mathrm{n}=11$ & $\mathrm{n}=12$ \\
\hline$C_{n}$ this work & $2.75 \times 10_{-3}$ & $-8.82 \times 10^{-4}$ & $2.39 \times 10^{-4}$ & $-6.75 \times 10^{-5}$ & $1.87 \times 10^{-5}$ & $-5.08 \times 10^{-6}$ \\
\hline
\end{tabular}

The correlation for the non-dimensionalised temperature development along the streamwise axis of the pipe is now found with:

$$
\Phi\left(\varepsilon_{R 2}\right)=\sum_{n=1}^{\infty} C_{n} \varepsilon_{R 2}
$$

where

$$
\mathrm{C}_{n}=-\frac{2}{\pi} \frac{h_{n-1}(0)}{n} \int_{0}^{\pi} \frac{[\sin (\phi)]^{1 / 3}}{\xi^{1 / 4}} d \phi
$$

\section{EXPERIMENTS}

Experiments of three independent research groups have been analysed for validation of the approach discussed above. All three experiments investigate water and nanofluid laminar flow in straight circular pipes. However, while the first two experiments $[10,15]$ employ a horizontal pipe, the third experiment [11] utilises a vertical pipe. Inner diameter and length of the pipes are $7.9 \mathrm{~mm} / 1,500 \mathrm{~mm}$ [10], $8.0 \mathrm{~mm} / 2,000 \mathrm{~mm} \mathrm{[15],} \mathrm{and} 4.5 \mathrm{~mm} / 1,050 \mathrm{~mm}$ [11], respectively. In all three cases the pipe is heated with a constant heat flux and the reference fluid is water. Data are taken in a comparable manner by thermocouples positioned along the axis of the pipes. Additionally, mass flux and inlet and outlet temperature are measured. In all three cases, Nusselt numbers are predicted from the obtained temperature distributions.

Nusselt number distributions of experiments by Buschmann [10] and Colla et al. [15] show definitive signs of mixed convection. This is true for reference fluid water, but also for nanofluids. Experiments by Colla et al. [15] reveals that onset of free convection and therewith departure from Nusselt number distribution from the analytical solution by Hsu [9] is significantly delayed by nanoparticles. The data by Rea et al. [11] do not show any signs of mixed convection, neither for water nor for nanofluid. However, it has to be clearly distinguished between the morphology of the secondary flow in horizontal and vertical pipe flow. In horizontal pipes, density gradients due to radial temperature gradients cause a pair of counter-rotating secondary vortices. These vortices transport colder fluid from the core of the flow to the inner wall of the pipe, which enhances heat transfer. Secondary flow in vertical 
pipes mainly means that the slope of the mixed convection profile is much steeper than the one of the pure forced flow profile and the profile forms a characteristic maximum near the wall [16].

\section{RESULTS}

In the following, experimental results of the three experiments [10,11, 15] are compared with the theoretical approach according to (10). First, data of the reference fluid water area analysed. In a second step, some data obtained with nanofluids as working fluid are discussed. Third, conclusions are drawn.

Figures 2 and 3 show the non-dimensionalised temperature distribution $F\left(e_{R 2}\right)$ according to eqns (3) and (10). All three plots show correlation (10) as a full line. Dashed lines indicate a departure of $\pm 10 \%$ from this curve. Two vertical red lines indicate the validity of the original approach by Hieber and Sreenivasan [14] (left line $\varepsilon_{R 2}=0.913$ ) and the extension presented with this work (right line $\varepsilon_{R 2}=2.361$ ).

\subsection{Vertical and horizontal pipe flow}

Experimental results for horizontal pipe flow by Buschmann [10] and Colla et al. [15] are compiled in Fig. 2. Data with high $\varepsilon_{R 2}$-values reaching well into the extended region are shown in the right plot. Note that the horizontal axis of the diagrams are logarithmically scaled.
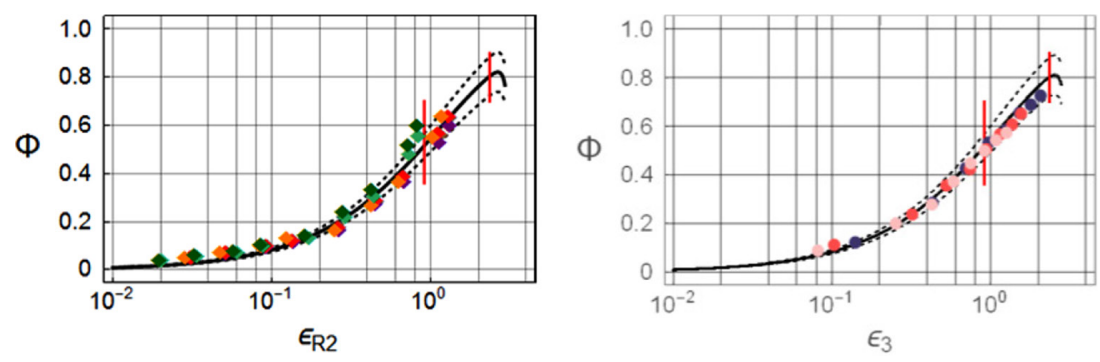

Figure 2: Experimental data for laminar flow in horizontal pipe with mixed convection $[10,15]$. Full curves indicate eqn (10), dashed curves a departure of $\pm 5 \%$, and dotted curves of $\pm 10 \%$.
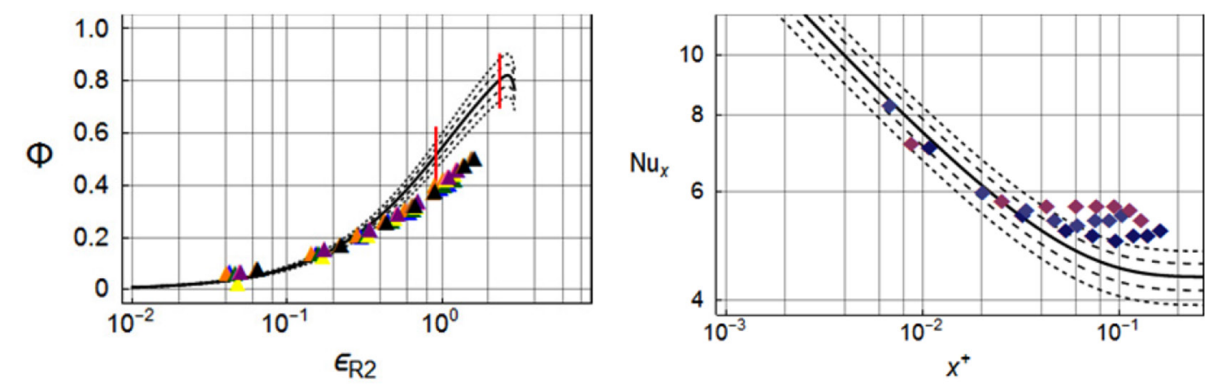

Figure 3: Experimental data for laminar flow in vertical pipe [11]. Curves as in Fig. 2. 
A reasonably good agreement is found between the data and theoretical results according to (10). The departure of the data from the theory by Siegel et al. [8] and Hsu [9] eqn (1) is obviously removed. The reason is simply that the non-dimensional streamwise coordinate $\varepsilon_{R 2}$ incorporates the Grashof number and therewith the intensity of free convection.

Figure 3 shows data from the vertical pipe flow experiment by Rea et al. [11]. The same data as in Fig. 1 (lower line) are shown. While a nearly perfect agreement with the theory without convection [8, 9] exists, a significant departure is found for the extended Hieber-Sreenivasan-approach. This is especially true for high $\varepsilon_{R 2}$-values. Reason is the different orientation of the pipe. Figure 1 indicates that there is no significant free convection. This is, of course, true, although the Grashof numbers for these experiments is not zero. The specific parameter combinations - $x / d_{i}, R e, P r, G r$ - lead to a nice collapse of the data, but do not correlate with eqn (10). The latter is again correct because neither the original approach by Hieber and Sreenivasan [14] nor its extension presented here is made for vertical pipes. Nevertheless, the collapse of the data indicates the chosen scaling might also be relevant for laminar flow in vertical pipes.

\subsection{Nanofluids}

Figure 4 shows data from nanofluid pipe flow experiments. The pipe is horizontally oriented and titania nanofluids of 1.0 vol. \% (left column) and of 2.5 vol. \% are employed. For details with respect to titania nanofluids refer to [15]. Heating power is always $100 \mathrm{~W}$ and inlet temperature at pipe entrance varies between $20^{\circ} \mathrm{C}$ and $40^{\circ} \mathrm{C}$.
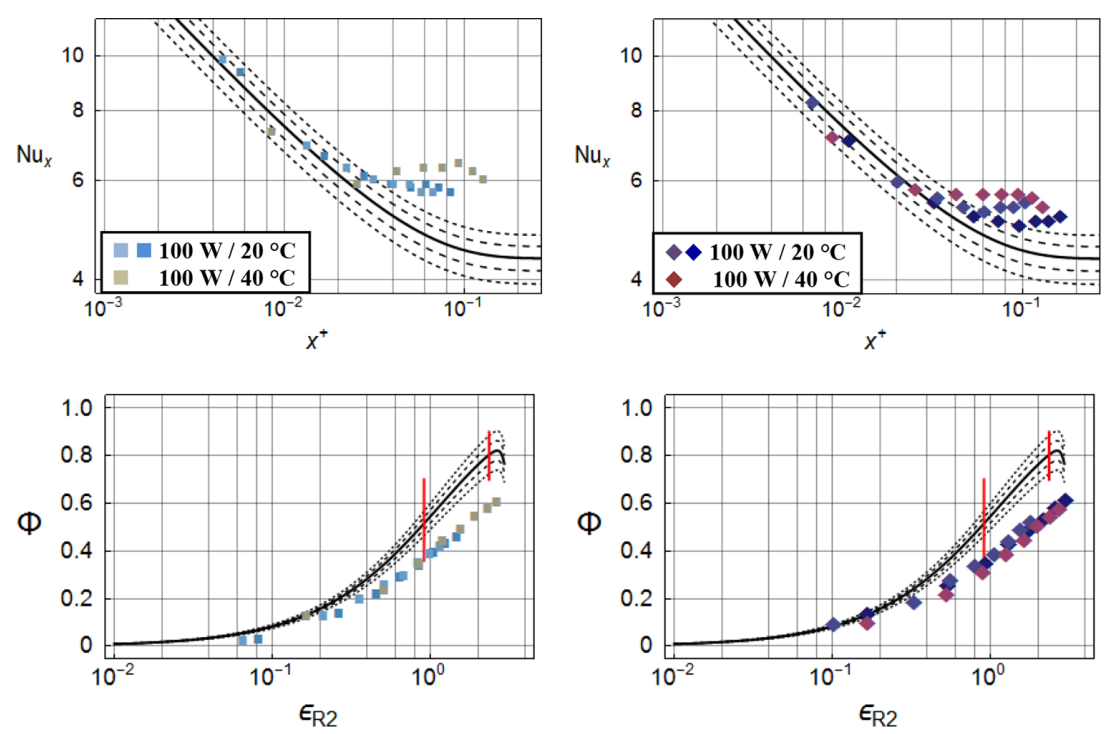

Figure 4: Experimental data for laminar nanofluid flow in horizontal pipe. Upper plots approach according to eqn (1) and lower plots according to eqn (10). Left row 1.0 vol. \% and right row 2.5 vol. \%. Curves in upper plots same as in Fig. 1 and in lower plots as in Fig. 2. Data are taken at CNR-Padova. 
Similar as with the data for pure water, the nanofluid results are in agreement with the classical approach $[8,9]$ as long as free convection can be neglected. Again, no collapse of data is obtained for $x^{+}$-values where secondary flow becomes relevant for heat transfer. Although, as pointed out by Colla et al. [15], the onset of the departure from eqn (1) is delayed in nanofluids compared to pure water, the scatter is still significant.

Scaling according to Hieber and Sreenivasan [14] leads to a striking collapse of the nanofluid data. As with water (Fig. 2 upper plots), the data form one single curve. This is also true for the two concentrations of nanoparticles (1.0 vol. \% and $2.5 \mathrm{vol} . \%)$ investigated. However, differently to water, this curve does not coincide with eqn (10). The departure is significantly larger than $\pm 10 \%$. Assuming that the character of the secondary flow is the same as in pure water flow, the found difference can only be explained by the nanoparticles affecting local heat transfer.

\section{CONCLUSION}

Nanofluids are attracting more and more attention. Proper test rigs and adequate testing strategies are needed to validate the potentials of these special fluids. Here, an analytical approach originally developed for laminar pipe flow with CWT boundary condition is extended and its applicability for laminar pipe flow with CHF boundary condition is verified. For that purpose, experimental data taken by three independent research teams were analysed.

The study shows that the approach by Hieber and Sreenivasan [14] can be extended up to 20th order employing computer algebra (MATHEMATICA 10.1). Scaling experimental data taken in horizontal laminar pipe flow with CHF boundary condition and significant secondary flow shows an excellent agreement with the obtained correlation. The incorporation of the Grashof number and therewith the consideration of free convection is key for this success. The situation is different for vertical laminar pipe flow. Although the data collapse nicely in one single curve, they do not coincide with the correlation found. The reason is simply that secondary flow in vertical pipe flow develops differently than in a horizontal one.

Experimental data from horizontal laminar pipe flow using titania nanofluids with two different concentrations are also analysed. It is found that again the data collapse in a perfect curve. Employing the correct effective thermophysical properties also removes variances following from the two different concentrations. However, the data do not match with correlation (10). The reason is the influence of nanoparticles which suppress free convection. To summarise, the new approach allows the distinguishing between effects following from free convection and those following from the influence of nanoparticles on heat transfer.

\section{ACKNOWLEDGEMENT}

The present work was done under grant MF090026.

\section{REFERENCES}

[1] Sergis, A. \& Hardalupas, Y., Anomalous heat transfer modes of nanofluids: a review based on statistical analysis. Nanoscale Research Letters, 6, pp. 1-37, 2011. http://dx.doi.org/10.1186/1556-276X-6-391

[2] Feja, S. \& Buschmann, M.H., Nanofluids - potentials and illusions, Split, Croatia, 12th International Conference on Simulation and Experiments in Heat Transfer and their Applications, Split Croatia, 2012. 
[3] Prabhat, N., Buongiorno, J. \& Hu, L.W., Convective heat transfer enhancement in nanofluids: real anomaly or analysis artifacts? Proceedings ASME/JSME 2011 8th AJTEC, Honolulu, Hawaii, USA, 2011.

http://dx.doi.org/10.1115/ajtec2011-44020

[4] Colla, L., Fedele, L. \& Buschmann, M.H., Laminar mixed convection of $\mathrm{TiO}_{2}$-water nanofluid in horizontal uniformly heated pipe flow. International Journal of Thermal Sciences, 97, pp. 26-40, 2015. http://dx.doi.org/10.1016/j.ijthermalsci.2015.06.013

[5] Colla, L., Fedele, L., Bobbo, S. \& Buschmann, M.H., Mixed convection in $\mathrm{TiO}_{2}$-water nanofluids. Fluids, 2015, in press.

[6] Graetz, L., Ueber die Wärmeleitfähigkeit von Flüssigkeiten. Annalen Physik, 18, pp. 79-94, 1883.

[7] Nusselt, W., Die Abhängigkeit der Wärmeübergangszahl von der Rohrlänge. Zeitschrift des Vereins Deutscher Ingenieure, 54, pp.1154-1158, 1910.

[8] Siegel, R., Sparrow, E.M. \& Hallman, T.M., Steady laminar heat transfer in a circular tube with prescribed wall heat flux. Applied Science Research, 7, pp. 386-392, 1956.

[9] Hsu, C.J., Heat transfer in a round tube with sinusoidal wall heat flux distribution. American Institute Chemical Engineering Journal, 11, pp. 690-695, 1965. http://dx.doi.org/10.1002/aic.690110423

[10] Buschmann, M.H., Thermal conductivity and heat transfer of ceramic nanofluids. International Journal of Thermal Science, 62, pp. 19-28, 2012. http://dx.doi.org/10.1016/j.ijthermalsci.2011.09.019

[11] Rea, U., McKrell, T., Lin- Hu, L.W. \& Buongiorno, J., Laminar convective heat transfer and viscous pressure loss of alumina-water and zirconia-water nanofluids. International Journal of Heat and Mass Transfer, 52, pp. 2042-2048, 2009. http://dx.doi.org/10.1016/j.ijheatmasstransfer.2008.10.025

[12] Morton, B.R., Laminar convection in uniformly heated horizontal pipes at low Rayleigh number. The Quarterly Journal Mechanics Applied Mathematics, 12, pp. 410-420, 1959.

http://dx.doi.org/10.1093/qjmam/12.4.410

[13] van Dyke, M., Extended stokes series: laminar flow through a heated horizontal pipe. Journal of Fluid Mechanics, 212, pp. 289-308, 1990.

http://dx.doi.org/10.1017/S0022112090001975

[14] Hieber, C.A. \& Sreenivasan, S.K., Mixed convection in an isothermally heated horizontal pipe. International Journal of Heat and Mass Transfer, 17, pp. 1337-1347, 1974. http://dx.doi.org/10.1016/0017-9310(74)90135-5

[15] Colla, L., Fedele, L. \& Buschmann, M.H., Laminar mixed convection of $\mathrm{TiO}_{2}$ water nanofluid in horizontal uniformly heated pipe flow. International Journal of Thermal Sciences, 97, pp. 26-40, 2015. http://dx.doi.org/10.1016/j.ijthermalsci.2015.06.013

[16] McEligot, D.M., McCreery, G.E., Schultz, R.R., Lee, J., Hejzlar, P., Stahle, P. \& Saha, P., Investigation off thermal-hydraulic phenomena in advanced gas-cooled reactors, INL/EXT-06-11801 MIT-GFR-042, 2006. 